\title{
Dossier
}

\section{El género en las políticas públicas: ¿una opción o una obligación? ${ }^{1}$}

Silvia A. Levín

Dra. en Ciencias Sociales (FLACSO Argentina)

Abogada (UNR)

Investigadora del Consejo de Investigaciones

de la Universidad Nacional de Rosario (CIC-CIUNR)

Directora del Centro de Investigaciones en Políticas Públicas,

Derechos y Gobierno (Facultad de Derecho - UNR)

E-mail:silvial@arnet.com.ar

1 A continuación se presentan las producciones correspondientes al Panel Especial "¿Política Social sin Género en Democracia?”, organizado por la Cátedra de Política Social II de la Licenciatura en Trabajo Social de la UNR, en el marco del X Congreso Nacional y III Congreso Internacional sobre Democracia, realizado del 3 al 6 de Septiembre 2012 en la ciudad de Rosario (Argentina) en la Facultad de Ciencia Política y Relaciones Internacionales de la Universidad Nacional de Rosario. Participaron del Panel Especial la Dra. Silvia Levín, la Lic. Mónica Bifarello, la Dra. Diana Maffía, la Dra. Laura Pautassi y la Lic. María Alejandra Ingaramo. 


\section{Introducción}

Durante las décadas de los noventa y del dos mil se ha afianzado en América Latina la idea de la estrecha vinculación existente entre política social, democracia y desarrollo, como resultado de estudios e investigaciones (UNESCO, 2003; PNUD, 2004; Faletto, 1993; etc.), a punto tal que hoy constituye una certeza que nos permite avanzar en sus postulados constitutivos centrales en términos sustantivos. La igualdad de género es uno de ellos.

El género hace referencia a una construcción social y cultural, que organiza nociones sobre aquello que sería "propio" de lo masculino y de lo femenino a partir de la diferencia sexual, no es natural y no afecta exclusivamente las decisiones individuales (Lamas, 1996). No se trata de una configuración identitaria individual de las personas, relacionadas con su modo de vivir la femineidad o la masculinidad, sino que influye de forma crítica en todas las relaciones sociales como la división sexual del trabajo, la distribución de recursos, el establecimiento de jerarquías, etc.

Subrayamos, entonces, que el género no constituye una categoría aislada ni una perspectiva suplementaria de otras para analizar los procesos políticos, sociales, económicos, culturales y simbólicos. Está inmerso en la trama de todas las relaciones sociales y en la medida que lo visualizamos podremos dar cuenta sobre la forma en que todos esos procesos estructuran esas relaciones (y viceversa) y actuar para transformarlas (igualdad/desigualdad/más igualdad); si lo negamos, también está, pero naturalizado y perpetúa las desigualdades. Desde que el género deja de considerarse como un tema específico del ámbito de las mujeres ${ }^{1}$, en las décadas de los 80 y 90 se produce

1 En la década del 70 comienza a tomar visibilidad la cuestión de las mujeres como actores excluidos de los procesos de desarrollo nacional y global (enfoque denominado "Mujeres en el Desarrollo" MED), durante las décadas de los 80 y los 90 surge una nueva perspectiva denominada "género en el desarrollo" (GED). La diferencia respecto al enfoque MED reside en que reconoce que los problemas de las mujeres no se originaban per se, sino que eran el resultado de determinada organización social y cultural, que otorgaba mayor autonomía y participación política y social a los hombres, lo cual conducía 
un viraje conceptual y político en la temática y pasa a ser entendido como una cuestión centrada en la sociedad y en el ser humano y que, por tanto, afecta a todos los ámbitos de la política y es un elemento fundamental para la consecución del desarrollo y la consolidación de la democracia.

La igualdad de género es un problema de Derechos Humanos desde el año 1948 (Declaración Universal de Derechos Humanos), fue ampliada en 1979 (CEDAW) y adquiere rango constitucional en nuestro país en 1994. Es tanto un principio marco, sustancial para la democracia y el desarrollo, como una obligación constitucional, que compromete al Estado y a la sociedad -a través de sus instituciones políticas, económicas y sociales- a garantizar el efectivo ejercicio los derechos y las prácticas. Las políticas públicas, y el conjunto de políticas sociales, son parte de ese proceso en tanto constituyen obligaciones estatales para el cumplimiento de los derechos y herramientas institucionales que deben respetar el marco conceptual y operativo de los Derechos Humanos a fin de viabilizar sus estándares (Pautassi, 2009:279). Una política social que no garantice derechos, entre ellos la igualdad de género, puede contribuir a una sobrevivencia circunstancial pero no a la integración estable y plena de las personas en el proceso de desarrollo y en el ejercicio de la democracia.

A nivel internacional, desde $1996^{2}$ los programas, fondos, gobiernos, proyectos, agencias, que integran el Sistema de Naciones Unidas asumen el compromiso de promover y garantizar la igualdad de género como eje transversal del desarrollo humano, incorporándolo de manera sistemática en sus Planes de Acción. Las múltiples acciones políticas llevadas a cabo en esta dirección y aprobadas por la Junta Ejecutiva de Naciones Unidas contribuyeron a su institucionalización. La vasta producción académica lograda acompañó, y es parte de este proceso político, al contribuir a cuestionar los problemas de

\footnotetext{
a la construcción de relaciones sociales de género atravesadas por jerarquías (PNUD, 2008:24).

2 Poco tiempo después de la IV Conferencia Mundial sobre la Mujer realizada en Beijing, en septiembre de 1995 , donde los 189 países que participaron se comprometen a garantizar la igualdad de género como eje del desarrollo humano y de consolidación de la democracia, Naciones Unidas comienza el proceso político de institucionalización de la igualdad de género.
} 
la desigualdad de género, a visibilizar sus lógicas y a exigir su tratamiento político como deuda de justicia, de democracia y de desarrollo. La Declaración del Milenio y los Objetivos de Desarrollo del Milenio aprobados por Naciones Unidas en el año 2000 constituyen un Programa Mundial mediante el cual los países, con los esfuerzos de los Estados y la sociedad civil, se comprometieron a promover activamente el ejercicio pleno de los Derechos Humanos y a mejorar las condiciones de vida de la población hacia el 2015. Uno de los Objetivos de Desarrollo del Milenio al cual se comprometieron 189 países del mundo -incluido Argentina- es promover la igualdad de género.

Alude a la necesidad de desterrar las diferencias entre varones y mujeres bajo un parámetro de dignidad básica común para todos. Permite ver y cuestionar la existencia de desigualdades en el ejercicio de derechos como parte de un proceso histórico, social y cultural e invita a identificar herramientas y recursos políticos y sociales para la igualdad (PNUD, 2008:10). Transcurre como proceso político, no como proceso meramente técnico, que promueve un nuevo modo de pensar y de actuar mediante el cual los estereotipos sobre mujeres y varones dejan lugar a una nueva filosofía que reconozca a todas las personas, independientemente de su sexo, como agentes imprescindibles para el cambio (PNUD, 1995). Por igualdad de género no se entiende que hombres y mujeres sean idénticos o sean lo mismo, sino pares en el status de derechos, responsabilidades y desempeño en el orden político, económico, social y cultural del desarrollo.

En consecuencia, la desigualdad es un problema de derechos humanos, de la democracia y del desarrollo. El problema de la desigualdad es el más grave que enfrentan las sociedades de América Latina, la región más desigual del mundo, y afecta tanto a aquella vinculación que mencionamos al inicio como a cada una de sus partes que intervienen. La envergadura de la desigualdad no comprende solamente carencias económicas y sociales, sino una compleja red de "desigualdades cruzadas” que se relacionan con el género, la ubicación geográfica, la raza o etnia, que ponen al individuo en desventaja y no operan nunca de manera aislada, sino que interactúan, crean ciclos 
de desventajas dinámicos, que se refuerzan mutuamente y se transmiten de una a otra generación (PNUD, 2005:66). La desigualdad es un fenómeno multidimensional, que enlaza privaciones y carencias en muchos aspectos del bienestar de manera interrelacionada y con intensidades diversas en cada uno, atravesadas todas por los factores de tiempo y espacio.

En función de ello, sostenemos, por un lado, que el enfoque de género y el de derechos humanos son constitutivos de la política social por su raíz institucional y su normatividad jurídica, lo cual otorga racionalidad y previsibilidad al tratamiento de los problemas complejos de su campo así como también certeza y legitimidad a las decisiones y acciones políticas orientadas a su resolución en clave de democracia y desarrollo.

El eje argumentativo principal se centra en mostrar, en primer lugar, que toda visión de política social que no contemple esos enfoques adolece de debilidad teórica y práctica porque desconoce la realidad social, la institucionalidad de la política y sus efectos en la estructura social. Nos apoyamos en evidencia empírica, sobre las tendencias de los últimos años, producida y reunida en documentos e Informes Regionales de la División de Asuntos de Género de la CEPAL (www.cepal.org.mujer) y del Observatorio de Igualdad de Género de América Latina y el Caribe (CEPAL, ONU, UNFPA, OPS) y en estudios teóricos específicos del campo de las políticas públicas.

En segundo lugar, a partir de la revisión crítica de la noción de "igualdad de oportunidades", anclada en la discriminación y que predomina en el campo de la política pública que atiende los problemas de la desigualdad de género, discutimos sobre las limitaciones que presenta para el tratamiento multidimensional de la desigualdad y, al mismo tiempo, sus efectos deficitarios en la democracia y el desarrollo. Como contrapunto para el debate introducimos las nociones de "igualdad estructural”, que trasciende la discriminación, y se centra en el no sometimiento ni exclusión y la de "igualdad de posiciones" que se ocupa de las desigualdades entre las posiciones en la estructura social sin poner directamente, sino indirectamente, el acento en las diferencias entre varones y mujeres. La intención es mostrar que una 
aplicación combinada de nociones de igualdad en el conjunto de las políticas públicas podría producir transformaciones en orden a las brechas entre desigualdades/democracia/desarrollo y no quedar restringida a las brechas entre desigualdad/ discriminación.

\section{Política Social, Democracia y Desarrollo}

El desarrollo sostenible, a través del crecimiento inclusivo, permanente y equitativo centrado en las personas, continúa siendo el objetivo principal de América Latina y por ello se renueva el compromiso de promoverlo en todos los niveles y dimensiones (Naciones Unidas, 2012:2). La institucionalización de la igualdad de género está directamente vinculada a la democracia en tanto ese proceso surgió, en la mayoría de los países, en medio de coyunturas democratizadoras de transición. Se articuló con la construcción de la democracia y esa interrelación permitió profundizar el reconocimiento de las mujeres como individuos (Amorós, 2007:60) $)^{3}$ protagonistas de ese proceso. Obviamente que esa institucionalidad democrática no ha garantizado por sí sola el desarrollo de la igualdad ni tampoco el reconocimiento de derechos o la ampliación de la ciudadanía de las mujeres, sino que fue necesario construir una compleja estrategia de interrelación de actores, intereses, normas y acuerdos a nivel internacional, regional, nacional y local.

Tanto el desarrollo como la democracia están directamente relacionados con la paridad de género (CEPAL, 2011:9), es decir con el logro de un tratamiento político y económico de hombres y mujeres como pares y para que ello suceda una de las condiciones indispensables es avanzar en la autonomía de las mujeres, en su vida pública y privada, como requisito para garantizar la igualdad efectiva de derechos. La autonomía (física, económica y en la toma de decisiones) es el pilar de la igualdad de género, en tanto capacidad de las mujeres

3 Siguiendo a Celia Amorós, entendemos que el estatuto de individuo habilita a que cada mujer marque su lugar diferencial, delimite su propio espacio y ejerza su propio poder. 
para desempeñarse y tomar decisiones libres de acuerdo a sus propias aspiraciones, deseos y expectativas de vida.

Hoy, en el marco de las condiciones materiales de América Latina, no se encuentra una explicación razonable a la desigualdad, a la falta de autonomía, a la mortalidad materna, al empleo precario o a la concentración del trabajo doméstico no remunerado en manos exclusivas de las mujeres (CEPAL, 2010a y 2011; Naciones Unidas, 1995). Por el contrario, estos resultados negativos se ubican mucho más cerca del plano de la injusticia; de la mala distribución del poder, los ingresos, la riqueza y el tiempo entre hombres y mujeres; y en la falta de reconocimiento de los derechos de las mujeres por parte de las élites políticas y económicas (CEPAL, 2011:9). Denotan, en general, la debilidad de la institucionalidad de género en los ámbitos gubernamentales, principalmente las dificultades políticas para reconocer y actuar en línea con el status de sujeto de derechos de las mujeres, así como también las dificultades culturales de la sociedad para producir los cambios democráticos necesarios.

Pareciera que, por un lado, transitan y avanzan los instrumentos normativos regionales, nacionales y los acuerdos internacionales plasmados en el corpus de derechos humanos que hacen al Estado de Derecho democrático y, por otro, su implementación que muchas veces tensiona o se contrapone con ese marco jurídico cuando, por ejemplo, se adoptan orientaciones asistencialistas en las políticas que la mayoría de las veces perpetúan los sesgos patriarcales, como por ejemplo, aumentar las responsabilidades del trabajo de cuidado no remunerado para las mujeres en detrimento del trabajo remunerado. Esta realidad, asociada a los cambios registrados y a los esperados, y esta institucionalidad reafirman que las políticas públicas y las políticas sociales son construcciones sociales, materiales y simbólicas.

Si bien no se desconoce que alcanzar la igualdad de género constituye un proceso, de la misma manera que consolidar un modelo de desarrollo en paridad, no es justo ni humano que el costo de ese tránsito siga recayendo en la vida (mortalidad materna, embarazo adolescente) y en el esfuerzo exclusivo de las mujeres (trabajo femenino no remunerado, doble y triple jornada laboral, división sexual del traba- 
jo, etc.) tal como exhiben los datos estadísticos. En este sentido, caben dos consideraciones respecto a las políticas públicas de igualdad: por un lado, se reconocen los avances buscados, y los no buscados, así como también nuevas demandas que coexisten con resistencias u oposición a esos avances por parte de algunos actores (por ej. los actores religiosos). Por otro lado, los resultados visibles de las políticas públicas, en términos generales y pese a los avances, muestran que aún no han logrado revertir la desigualdad y la discriminación (CEPAL, 2011:45). Muchas veces la retórica del discurso político favorable a la igualdad de género o a la promoción de derechos de las mujeres ha agotado la instancia de la intervención pública sin llegar a traducirse en políticas concretas.

En el contexto global y nacional de la reciente y progresiva revalorización del Estado se ha jerarquizado su papel respecto a las políticas públicas y, en particular, con la igualdad de género. En este punto, es preciso remarcar la necesaria corresponsabilidad de gobiernos, sociedades y familias para que asuman conjuntamente las exigencias de ese tránsito, de lo contrario no sólo el desarrollo se distorsiona sino también la democracia en sí misma, en tanto las mujeres, la mitad del país, ven restringido su desempeño pleno como ciudadanas, sujetos de derecho, es decir su inclusión política.

\section{Visiones de Igualdad: entre oportunidades, posiciones y estructura}

La evidencia empírica producida en la última década en la región ${ }^{4}$ da cuenta de que, a pesar de los esfuerzos normativos del campo de los Derechos Humanos y de los avances alcanzados con la implementación de políticas públicas de igualdad de género ancladas en la igualdad de oportunidades y de trato entre hombres y mujeres, no se

\footnotetext{
4 Se utiliza principalmente información producida por el Observatorio de Igualdad de Género de América Latina y el Caribe (OIG). Véase en línea:www.cepal.org/oig; CEPAL (2011), Informe anual El salto de la autonomía de los márgenes al centro. Observatorio de igualdad de género de América Latina y el Caribe, CEPAL, septiembre, Chile; CEPAL (2012) El Estado frente a la autonomía de las mujeres, Colección la Hora de la Igualdad, julio, Chile.
} 
ha logrado aún revertir la desigualdad y la discriminación en términos de resultados (CEPAL, 2011:45).

Estas políticas de igualdad de género, coordinadas y dirigidas por los mecanismos institucionales para el adelanto de la mujer tuvieron como punto de partida la Plataforma de Acción de Beijing aprobada en 1995 en la IV Conferencia Mundial sobre la Mujer, los consensos regionales posteriores y los Objetivos de Desarrollo del Milenio. Actualmente se encuentran en un punto de inflexión, que deriva tanto de su desarrollo como de su retroceso así como también de los cambios acontecidos en el contexto político e institucional, lo cual permite revisar tendencias generales sobre los diferentes tópicos que hacen a la igualdad. Es paradójico pero, como se advierte, por una parte, están los efectos buscados, y los no buscados, en los avances de la igualdad de género -que generan nuevas demandas junto a resistencias e incluso oposiciones- $y$, por otra parte, los resultados poco visibles de esas políticas que, en general, no han logrado cambios sustantivos en orden a la desigualdad y a la discriminación.

En este apartado pretendemos, justamente, poner en discusión la noción de "igualdad de oportunidades y de trato" que se aplica y predomina -en nuestro país y en la región- en el campo de las políticas públicas de igualdad de género, con sus diversos matices, para contrastarla con otras visiones de igualdad que ofrece la literatura con el propósito de establecer hasta qué punto el enfoque de oportunidades es capaz de contribuir a enfrentar y/o disminuir las desigualdades cruzadas que nos afectan.

Nuestra hipótesis es que resulta necesario trascender el enfoque de la "igualdad de oportunidades" mediante visiones que contemplen las oportunidades, pero que la superen, profundicen en decisiones y acciones respecto al tratamiento de las otras aristas de la desigualdad con visos de transformación estructural. Aquella visión cumplió su ciclo en la lucha contra las desigualdades de género desde que en coyunturas democratizadoras de transición comienza a institucionali- 
zarse el enfoque de género en la mayoría de los países de la región y se mantiene hasta la actualidad ${ }^{5}$. En ese escenario, fue crucial vincular discriminación con desigualdad y, al mismo tiempo, viable articular la equidad de género con la construcción de la democracia y a partir de allí reconocer a las mujeres como sujetos protagonistas de ese proceso.

Hoy constituye una certeza que la democracia y también el desarrollo necesitan de la paridad de género, es tanto una responsabilidad política como un deber legal, a pesar de ello, sabemos que hay reticencia política en algunos escenarios para garantizarla. Las desigualdades entre varones de mujeres son política, social, cultural y económicamente inadmisibles, el consenso internacional de Derechos Humanos lo dejó plasmado. Pero en las últimas dos décadas las desigualdades siguieron creciendo y se profundizaron, no sólo las de género, sino que se entrecruzaron otras y la "igualdad de oportunidades" ya no puede por sí sola afrontarlas, ni incidir de manera suficiente para reducir o detener las propias del campo de género. Por ejemplo, la visión de igualdad de oportunidades resulta insuficiente para visibilizar y actuar sobre los problemas del cuidado o sobre los de violencia. No es un propósito del enfoque ocuparse de las posiciones sociales de varones y mujeres en la estructura social o de las brechas entre las posiciones de uno u otro o de las dificultades que crean desigualdades en y entre las posiciones. Es necesario adoptar una visión de igualdad que supere la discriminación y pueda actuar en la desigualdad y transformarla en el marco de la complejidad de las desigualdades cruzadas.

$\mathrm{La}$ "igualdad de oportunidades y de trato"6, que sustenta las políticas de igualdad de género, constituye una de las visiones de igualdad, que se impuso en la mayoría de los países por sobre otras, como

5 Los Planes y/o Programas de igualdad de oportunidades (PIO) implementados y vigentes en la actualidad en buena parte de los gobiernos locales, coordinados a través de Áreas o Direcciones de la Mujer o de Género creadas desde fines de los ochenta, constituye evidencia en esa dirección. El municipio de Rosario fue uno de los pioneros en la implementación de esta línea de política pública en Argentina. El Primer PIO comprende el período 2001-2004, el segundo PIO 2005-2009 y actualmente está en marcha el tercer PIO 201-2015.

6 Surge del modelo de liberalismo de Estados Unidos. 
la "igualdad de posiciones" (Dubet, 2012) o la igualdad sustantiva o también denominada "igualdad estructural" (Saba, 2005). Esa decisión no sólo tiene connotaciones teórico conceptuales específicas, sino también efectos prácticos, en términos de políticas sociales y de programas políticos. Esto es, optar por una u otra concepción de igualdad no implica sólo elegir entre uno u otro enfoque teórico, sino asumir distintas maneras de concebir la realidad y de actuar en ella. Pero, por otra parte, y tal vez sea lo más importante, la decisión acerca de cuál o cuáles concepciones adoptar está directamente relacionada con el tipo de problemas y su magnitud en la realidad que se deba enfrentar. América Latina es la región más desigual del mundo y las desigualdades son "cruzadas" (CEPAL, 2005), no sólo económicas y sociales como buena parte del mundo académico y, en particular, especialistas en política social aún afirman, por tanto, la igualdad capaz de contrarrestar este drama deberá contemplar necesariamente esa complejidad para transformarla.

Cada concepción de igualdad produce movimientos diferentes en la estructura social, que pueden favorecer, sostener o perjudicar a sectores, grupos e intereses distintos, y hasta constituir actores disímiles. No obstante, unas y otras, parten de reconocer un problema común: cómo acortar la brecha entre las igualdades fundamentales y las desigualdades sociales reales y, en ese marco, un propósito común: intentar reducir esas desigualdades, al menos para volverlas más justas o aceptables (Dubet, 2012:43).

Alcanzar o no mayores niveles de igualdad, o reducir o no las desigualdades es un problema de justicia social. De modo tal, que la concepción de igualdad que se adopte se traslada también a los criterios de justicia que resuelven los conflictos sociales y la política judicial, entonces, puede contribuir a realizar justicia o a profundizar o sostener injusticias (Levín, 2012:13).

7 El estudio de sentencias judiciales de la Corte Suprema de Justicia de la Provincia de Santa Fe ha permitido reunir evidencia sobre los efectos que produce en un mismo caso la aplicación o no de una concepción de igualdad que trascienda las oportunidades, como la "igualdad estructural" o sustantiva, en conflictos anclados en desigualdades cruzadas (económico sociales y de género). La diferencia, por ejemplo, en uno de los casos osciló entre condenar a prisión perpetua a una persona (en primera instancia y en la Cámara de Apelaciones al no valorar la posición social y condicionamientos de género de la imputada) a liberarla de todo cargo. En efecto, el fallo de la Dra. Gastaldi, Corte Suprema de 
La "igualdad de posiciones" y la "igualdad estructural", versiones más sociológicas de la idea de igualdad, tienen en común que ponen el acento en las posiciones o espacios sociales que ocupan los individuos -sean hombres o mujeres, minorías étnicas, etc.- ocupan -como parte de una clase social o grupo o colectivo- en la estructura social. La "igualdad de posiciones" se preocupa por reducir las desigualdades de ingresos, de condiciones de vida, de acceso a servicios, de seguridad, que están asociadas al nivel de formación, sexo, edad, trayectoria profesional, etnia, etc., que cada individuo tiene en la sociedad. Su propósito es achicar la brecha entre las desiguales posiciones sociales, ajustar la estructura social, pero sin poner el acento directamente en la movilidad social ${ }^{9}$. Es en el terreno de las condiciones de trabajo y de los salarios donde se constituyen y desde donde se reducen las desigualdades sociales. La lucha contra las desigualdades se focaliza en la esfera del trabajo y los sindicatos son actores de ese proceso. Las críticas que se hacen a la concepción de "igualdad de posiciones" respecto a la posibilidad real de concretar la igualdad son varias (Dubet, 2012:44), entre ellas, adoptar rasgos próximos a un Estado de Bienestar, corporativista, para concretar sus aspiraciones y promover una solidaridad social ciega a las discriminaciones.

La "igualdad estructural”, por su parte, agrega al concepto de posiciones que toda consideración que se haga sobre una persona debe referirse no sólo a ella en términos individuales, sino también y sobre todo, como parte de un colectivo o de un grupo con el cual se identifica y comparte su posición social que ha sido sistemáticamente excluido o sometido. Esa identidad y esa pertenencia se constituyen en torno a varias condiciones sociales comunes, entre ellas, por ejem-

\footnotetext{
Justicia de la Provincia de Santa Fe, al que adhiere el cuerpo, pone énfasis en la argumentación en la posición social de pobreza extrema y en los graves condicionamientos y sometimientos de género -violencia sexual, embarazos no deseados, privación ilegítima de la libertad- que pesaban desde niña en la historia de la acusada. Para mayor información ver Levín (2012).

8 Impulsada por el movimiento obrero y por los ideales socialdemócratas.

9 Por ejemplo, no se trata de prometer a las mujeres que tendrán tantas oportunidades de tener autonomía económica como los varones, sino de reducir la brecha en las condiciones de vida y de trabajo entre varones y mujeres. No se pone el énfasis en que las mujeres puedan ocupar el mismo trabajo de mercado remunerado que los varones, sino en que el acceso al trabajo de mercado remunerado sea igual para varones y para mujeres.
} 
plo, pertenecer al colectivo de mujeres, y tienen implicancias en las prácticas políticas, económicas y sociales dirigidas a los miembros de ese colectivo en el marco del conjunto social.

Por otra parte, esta visión completa y trasciende la concepción de la "igualdad de oportunidades" al incorporar en su consideración el problema de la discriminación. Busca evitar no sólo la "ceguera" de la discriminación sino también la "ceguera" a toda valoración social sobre las posiciones en que se desenvuelven las relaciones sociales existentes, enmarcadas en "datos históricos y sociales" tales como jerarquías, conflictos de poder e intereses, explotación, subordinación (Saba, 2005:126). La desigualdad implica para esta visión, sometimiento y exclusión sistemática de amplios sectores por otros sectores de la sociedad. Según el autor, es esta visión de igualdad estructural o sustantiva, la que está plasmada en nuestra Constitución Nacional de 1994 (arts. 16 y 75 inc. 23), a pesar de que no es la que se aplica mayoritariamente, sino que predomina la visión liberal de las oportunidades.

La visión de oportunidades, que tiene su raíz en un enfoque individualista y liberal de la igualdad, entiende a ésta como "no discriminación y trato igual en las mismas circunstancias" ${ }^{10}$, aspira a luchar contra las discriminaciones que obstaculizan la realización del mérito para acceder a posiciones desiguales pero como resultado de una competencia equitativa entre individuos que se enfrentan para ocupar puestos sociales. Aquí, las desigualdades sociales son entendidas como obstáculos que se oponen a la competencia equitativa (Dubet, 2012:46). No se discuten las desigualdades de y entre las posiciones sociales (jerarquías ocupacionales, diferencias de ingreso, etc.) sino las discriminaciones (sexo, edad, raza, etc.) que obstaculizan a cada cual a acceder a esos puestos. Su lógica se apoya en una visión más descontextualizada de la situación de cada individuo, a diferencia de la "igualdad estructural" que se sostiene en una visión contextualiza-

\footnotetext{
10 "Tratar igual" no significa para nuestra Constitución Nacional (art.16) tratar a todas las personas como si fueran lo mismo. El Estado tiene facultades para tratar de modo diferente a las personas siempre que esa diferencia se funde en un criterio justificado o razonable. Justamente, la determinación de lo que es "razonable" constituye el tema de fondo de la discusión sobre la noción de "igualdad estructural".
} 
da (o sociológica) de una realidad social más amplia, que contempla la pertenencia del individuo a un grupo que se encuentra sometido a tratos o prácticas sociales como consecuencia de ser parte de ese grupo (Saba, 2005:138). Por otra parte, requiere siempre de una suerte de "intención de discriminación", pero positiva, reconocida a partir de la irrazonabilidad del criterio utilizado para producir la diferencia.

Se preocupa menos por lograr que las desigualdades de posición sean limitadas en las sociedades, que porque las personas puedan acceder a todas las posiciones sociales en función de sus proyectos y sus méritos. Por ejemplo, en este modelo de oportunidades, no se pone tanto el acento en buscar transformar las jerarquías que existen en las actividades profesionales y en las diferencias en los ingresos entre hombres y mujeres, sino que las mujeres tengan igual presencia en todas las posiciones sociales (Dubet, 2012:46). Compromete siempre mucho más la responsabilidad de cada individuo que la de toda la sociedad, porque es su mérito el que puede optimizar sus oportunidades. En este enfoque, según Dubet, las representaciones de la sociedad cambian, las clases sociales (enfoque de posiciones) son sustituidas por grupos que se definen sobre la base de las discriminaciones que sufren y, como se trata de adscripciones negativas, para afirmarse recurren a exigencias de reconocimiento que transforman el estigma de la diferencia en características positivas que profundizan la diversidad en la sociedad y se arriesgan los modos de representación política.

En general, no se ha intentado coordinar una combinación coherente de distintas visiones de igualdad, en tanto ninguna por sí misma parece poder abarcar y enfrentar la complejidad de la desigualdad para reducirla o resolverla. Tampoco se ha buscado, por ejemplo, establecer un orden de prioridad en la aplicación de concepciones, de manera tal que permita conjugar estratégicamente los atributos positivos de unas y otras en orden a la realización de una compleja pero necesaria mayor igualdad. 


\section{¿Qué igualdad puede atender la desigualdad en la política pública?}

Durante décadas las políticas macroeconómicas neoliberales promovieron la reducción del papel del Estado, la desregulación de los mercados financieros y de trabajo y concibieron a las políticas sociales como compensatorias de las dinámicas excluyentes propias de ese modelo. En este marco las políticas públicas de género, que requieren de Estados democráticos y rectores por definición, han avanzado a contrapelo de esa realidad política, económica y social. Mientras se reconocían los derechos de las mujeres, en el plano de los Derechos Humanos, se debilitaba la institucionalidad del Estado y los objetivos de igualdad se subordinaban a los de crecimiento concentrado (CEPAL, 2012:13).

Como consecuencia de ese escenario, la igualdad de género, en general, ha operado mucho más en la periferia de los procesos de reforma que en los núcleos de decisión del conjunto de las políticas públicas. Por otra parte, el enfoque de género ha tenido mayor incidencia en el campo de las reformas sociales y menor en el de las grandes reformas estructurales, económicas y de administración del Estado. Al mismo tiempo, se advierte una preocupación por evaluar hasta qué punto las políticas sociales de género superan el perfil asistencialista del corto plazo y fortalecen la ciudadanía de las mujeres (CEPAL, 2003:10).

No obstante ello, las políticas de igualdad se han sostenido sobre la base de la voluntad política dado que no cuentan con los recursos necesarios y suficientes -políticos, financieros y técnicos- para cumplir las funciones asignadas en cada país. En los países en los que se han logrado consolidar alianzas con el sistema político y con el movimiento feminista -y otras organizaciones sociales-, los resultados fueron más exitosos en términos de instalar la agenda de género (CEPAL, 2011:46) y concretar algunos cambios sustantivos ${ }^{11}$.

11 Un claro ejemplo de resultados de políticas públicas con impacto de género a partir del entramado de alianzas entre el sistema político, el movimiento feminista, organizaciones sociales diversas y sectores del mundo académico fue la aprobación en el Congreso de la Nación, en el año 2002, de la 
La profundidad de las desigualdades, rasgo distintivo de este continente desde el siglo XX (O’Donnell, 1999) y, de algún modo, el grado de aceptación de su existencia por diversas sociedades nacionales, así como también la cultura política democrática y los lineamientos hegemónicos que orientan las estrategias de desarrollo, entre otros, han condicionado el curso y el sentido del proceso de institucionalización de las políticas de igualdad.

La supuesta preocupación pública por la protección de los sectores vulnerables, pobres e indigentes y en situación de exclusión social, fue canalizada en el proceso de reformas socioeconómicas neoliberales pro-mercado a través de una amplia gama de programas de combate a la pobreza que en su mayoría reforzaron las desigualdades de género al promover la maternidad y el cuidado como responsabilidades exclusivamente femeninas. Estos convivían en materia social, simultáneamente, con el recorte de las políticas sociales, ya sea privatizadas o con criterios restrictivos para la selección de beneficiarios. La protección social fragmentada y estratificada fue su característica principal y el rasgo heredado que aún persiste y cuesta revertir.

Las estrategias sociales, denominadas transferencias condicionadas de ingresos (TCI) o de rentas concentran hoy, aunque surgieron a mediados de 1990, buena parte de la intervención pública orientada a atender los riesgos sociales de las desigualdades de los países de la región (pobreza, desigualdad socioeconómica, generacional, de género, étnica, racial, etc.). Si bien se las ubica, por un lado, como alternativas públicas con potencialidad de convertirse en un eslabón específico y permanente de los sistemas de bienestar incompletos, por otro lado, se advierte también sobre las limitaciones que comparten con los programas que las antecedieron (Midaglia, 2012:80).

En rigor, tanto el repliegue del Estado como el recorte en los gastos sociales en los noventa fueron suplantados por diferentes programas de combate a la pobreza extrema, sostenidos con financiamiento internacional que buscaron posponer el conflicto distributivo 
y reemplazar las políticas sociales más universales -líneas de acción sustentables política, institucional y financieramente- por proyectos formulados sobre la base de parámetros de medición y evaluación (Midaglia, 2012:81). Obviamente que el avance técnico en el diseño de nuevas propuestas de protección social como las TCI es meritorio, porque se advierte un cierto giro político en el tratamiento de la pobreza y, en particular, la asunción por parte del Estado de una cuota de responsabilidad al transferir recursos a ciertos sectores sociales vulnerables provenientes de las arcas públicas. No obstante, ello no reemplaza la lógica política e institucional y el impacto social de las acciones públicas estables, con garantías de financiamiento específico del presupuesto público nacional. Como señala la autora, estas iniciativas nuevas de tratamiento de la vulnerabilidad se transformaron en el núcleo inicial moderno, e inestable, del componente de asistencia que se constituye en la región.

En Argentina, la asignación universal por hijo (AUH) es la última iniciativa pública, creada por decreto del Poder Ejecutivo Nacional del año 2009, que se ubica en este registro. Si bien no nos vamos a detener en su consideración particular porque no constituye el objeto de este trabajo, nos interesa traerla a colación como la forma más reciente de TCI de amplio alcance -dirigida especialmente al tratamiento de la vulnerabilidad y la pobreza- cuyos titulares beneficiarios son en su mayoría mujeres (ANSES, 2011) y que busca insertase en un esquema general de protección social del país, que convive con otras políticas asistenciales y con políticas sociales ancladas en derechos.

Los estudios e investigaciones del campo académico, informes regionales e informes de organizaciones sociales y de mujeres, son coincidentes en afirmar que todos los programas TCI, al igual que la AUH, tienen en común la particularidad de reforzar y reproducir las relaciones tradicionales de género centradas en la idea de que el cuidado es una responsabilidad individual, no social, exclusiva de las mujeres y no compartidas (miembros de la familia y/o progenitores), profundizando así las desigualdades entre varones y mujeres en orden a ejercitar iguales derechos relativos a tiempos, ingresos y desempeño 
en el trabajo productivo remunerado y en el trabajo reproductivo no remunerado ${ }^{12}$.

Las particularidades de la AUH en Argentina están asociadas al instrumento utilizado para las TCI del ámbito de la Seguridad Social, como son las asignaciones familiares. Estas asignaciones se encuentran consolidadas en el ámbito laboral formal como beneficios provenientes de políticas contributivas y, paralelamente, comprenden también beneficios, derivados de políticas no contributivas, para la población con ingresos insuficientes y del sector laboral informal. Este arreglo institucional permite establecer un nexo, aunque débil, con el esquema general de protección social consolidado en el país. No obstante ello, esta vinculación con la Seguridad Social lleva implícita dificultades, una de ellas es que los recursos asignados a la financiación de la AUH no son propios, sino que pertenecen al Fondo de Garantía de Sustentabilidad de la ANSES provenientes del sistema contributivo lo cual condiciona considerablemente esos recursos y la viabilidad de la política.

La literatura específica que aborda el estudio de estas nuevas estrategias sociales de protección (Serrano, 2005; Lomelí, 2008; Arcidiácono, 2012; entre otros) en el marco de un balance, advierte sobre sus rasgos positivos y sobre sus rasgos negativos. En esta línea, algunos autores identifican su carácter dual en términos de orientaciones políticas respecto al bienestar, al combinar en su interior aspectos liberales y aspectos intervencionistas (Midaglia y Silveira, 2011; Midaglia, 2012). Entre los aspectos liberales se señalan: la focalización de su operatoria; el cumplimiento de obligaciones para percibir el beneficio lo que debilita su condición de derecho; el enfoque de pobreza asociado a capital humano y disociado del problema de la distribución de riqueza; y el bajo financiamiento asignado para su

12 Para mayor información consultar: Informe alternativo de Organizaciones de la sociedad civil para presentar a la CEDAW, Resumen Ejecutivo, "Derechos Humanos de las Mujeres: asignaturas pendientes del Estado Argentino”, CELS, CLADEM, FEIM, CEDES, INSGENAR, Católicas por el derecho a decidir, Buenos Aires, 2010; Goren Nora (2011) "La AUH ¿conquista de nuevos derechos? ¿viejas o nuevas identidades femeninas?, ponencia presentada al $10^{\circ}$ Congreso Nacional de Estudios del Trabajo, Buenos Aires; Pautassi L y Zibecchi C. (2010) La provisión de cuidado y la superación de la pobreza infantil. Programas de transferencias condicionadas en Argentina y el papel de las organizaciones sociales y comunitarias. Serie Políticas Sociales 159, CEPAL, Santiago de Chile. 
puesta en práctica ${ }^{13}$. Entre los aspectos intervencionistas de las TCI se mencionan: la obligación asumida por el Estado de garantizar un mínimo de ingresos y bienes sociales esenciales -educación, salud-; el desarrollo de sistemas de información social para facilitar el acceso a los servicios públicos y la mejora de las funciones regulatorias en relación a la oferta social.

En definitiva, si bien hay indicios de un cambio de rumbo político en el tratamiento público de las desigualdades vulnerables éste es aún incompleto, debe profundizarse a fin de poder integrar con un formato institucional sólido el conjunto del esquema de bienestar social del país. Sin embargo, este avance en el diseño de instrumentos de política pública no tiene su correlato en términos de reducción de las desigualdades económicas y sociales. Si bien hay evidencia de que la AUH produjo un aumento en los ingresos económicos de los hogares, no ha logrado aún impactar en las brechas de desigualdad.

Estos parámetros de incidencia social dan cuenta de que esta medida no puede agotar por sí misma la batalla contra la desigualdad y la vulnerabilidad, sino que es necesaria su articulación con políticas de rango universal. Las "desigualdades cruzadas" (género, raza, etnia, territorio, etc.) exigen un tratamiento multidimensional que enlace todas las aristas de la desigualdad dado que su dinámica de desenvolvimiento no admite fragmentación ni disociación en y entre concepciones y prácticas políticas institucionales. La pobreza y la vulnerabilidad, como fenómenos derivados de la desigualdad, son también en sí mismos multicausales.

Si las prácticas políticas institucionales intervienen sobre la desigualdad desconociendo la problemática de género que subyace en su raíz o, a la inversa, si las políticas de igualdad de género hacen foco en la discriminación, a través de las oportunidades, pero no inciden en los condicionantes económicos, sociales y culturales que interfieren en las posiciones de hombres y de mujeres y en la distancia que existe entre las posiciones de unos y otros en la estructura social; en

13 Se lo calcula en relación al PIB anual, siendo Argentina y Uruguay los países que más fondos destinan a estas iniciativas, entre $0,60 \%$ y $0,80 \%$ en 2002 y 2008 respectivamente. 
ambos casos el resultado final será siempre más desigualdad. Pero valoremos las situaciones en base a ejemplos ilustrativos de ambas opciones políticas. En el primer caso, señalamos que la AUH no interviene para modificar los patrones tradicionales de género, sino que los refuerza, esto implica, por tanto, que si el cumplimiento de las contraprestaciones en salud y educación de los hijos recae exclusivamente sobre la mujer, aumenta su carga de trabajo no remunerado y disminuye su tiempo real de trabajo remunerado. A estas injustas desigualdades se suma el déficit público respecto al cuidado, dado que dichos programas no incorporan servicios que contribuyan al trabajo de cuidado (de niños, ancianos, discapacitados, etc.) para garantizar el derecho al trabajo remunerado de la mujer.

Al mismo tiempo, esas contraprestaciones son estigmatizantes para las madres porque deben "certificar" las condicionalidades de salud y educación sin garantías institucionales adecuadas y suficientes (instituciones escolares suficientes y accesibles geográficamente, instituciones de salud gratuitas) para que lo puedan efectuar (Pautassi, 2010). Por otra parte, si consideramos que el alto porcentaje de beneficiarias son mujeres, y que en contextos de pobreza urbana la mayoría de los hogares tienen jefatura femenina, podemos concluir que el déficit de género en la AUH compromete seriamente los resultados de la política además de no garantizar que esos hogares superen en un corto o mediano plazo la indigencia o la pobreza.

En el segundo caso, no produce los mismos efectos en términos de igualdad y bienestar una política de igualdad de oportunidades orientada a promover emprendimientos productivos liderados por mujeres en situación de pobreza que garantizar a través de una batería de políticas sociales institucionalizadas la mejora de salarios, de las condiciones de vida estructurales y medidas públicas de provisión de cuidados en barrios vulnerables de un determinado territorio.

Si bien una u otra opción de política pública dan muestra de no poder enfrentar adecuadamente el problema de la desigualdad, no pueden reducirla ni resolverla, tal vez una combinación apropiada y estratégica de atributos de unas y otras pueda contribuir a desarrollar otra institucionalidad de prácticas políticas, que, obviamente tendrán 
que estar en sintonía con el modelo económico y de desarrollo. En esa línea continuamos nuestra argumentación.

Si encarar el fenómeno de las desigualdades constituye una complejidad de la envergadura señalada, en términos cuantitativos y cualitativos, la concepción de igualdad que se adopte para encauzar propósitos de acción pública con capacidad de transformación social en el marco de estrategias de desarrollo, no constituye un tema menor, sino que debe estar a la altura, teórica-conceptual y práctica-operativa de esos acontecimientos tal como lo señalamos en anteriores apartados.

La concepción de "igualdad estructural" o igualdad sustantiva contempla las oportunidades pero las trasciende, con el convencimiento de que si sólo se atienden los componentes discriminatorios (sexo, edad, color de la piel, raza, religión etc.) no se garantiza la igualdad, sino que es necesario además sumar datos sociológicos e históricos, es decir patrones sociales, económicos y culturales de pertenencia, que identifiquen al individuo pero como parte de grupos o colectivos de personas que comparten su historia social. De ese modo, podrán establecerse parámetros de sometimiento, de subordinación y/o explotación sistemática que algunos grupos ejercen sobre otros. Esta visión sociológica de la igualdad permite centrar el problema en el individuo, por tanto controla la discriminación, pero lo considera insuficiente y avanza en su pertenencia social, a grupos o colectivos, con los cuales se identifica en perspectiva histórica, en sus posicionamientos socioeconómicos y culturales.

No adhiere, entonces, a un trato segregacionista y excluyente tendiente a consolidar grupos marginados. Para esta visión para garantizar la igualdad es admisible, tratar de modo diferente a las personas de acuerdo con el grupo (personas de un determinado sexo) al que pertenecen, privilegiando, por ejemplo, a las mujeres sobre los varones en el acceso a puestos jerárquicos de empleos en una empresa, en una situación sistemática de exclusión de aquéllas a la ocupación de ese tipo de puestos. En esta línea caben las acciones afirmativas, pero 
resultan controvertidas, en cambio, para la concepción de igualdad de oportunidades ${ }^{14}$.

La "igualdad de posiciones" provee la posibilidad de un ajuste en la estructura social, busca achicar brechas de desigualdad entre las posiciones de unos y otros, por tanto favorece la cohesión social y, al mismo tiempo, la autonomía de las personas. Fortalece el sistema de derechos y obligaciones, promueve el sentido de lo común más que el de competencia. No se opone a la igualdad de oportunidades, sino que la posibilita porque permite el desarrollo del mérito y la circulación en las distintas posiciones de la estructura social. En todo caso, lo importante de este enfoque es que permite enlazar las desigualdades, no disociarlas, y contribuye a tratar el fenómeno más allá de la discriminación.

En síntesis, reducir y/o resolver las desigualdades no es un problema de voluntad sino de justicia. Afirmar que un problema pertenece al orden de la justicia implica que no son razones -fundamentos legítimos justificados conforme a derecho- las que producen o sostienen esas desigualdades sino arbitrariedades -de poder, de jerarquías, de sometimiento- que violentan y frenan los procesos de desarrollo y de integración; que benefician a unos a costa de perjudicar a otros. Se ha comprobado que el crecimiento económico por sí solo no es suficiente para atender este fenómeno (Salvia, 2011:33), si bien hubo en Argentina en el año 2009 una recuperación importante de los sectores sociales medios y un aumento considerable del gasto social, la economía creciente no pudo superar la exclusión estructural y las des-

\footnotetext{
14 En nuestro país no hay demasiados antecedentes que muestren debates, cuestionamientos o conflictos entre concepciones de igualdad, en particular sus derivaciones en la aplicación de enfoques diversos y sus efectos políticos y sociales. En el campo de la justicia y del derecho se están realizando algunos estudios apoyados en la teoría política y en la filosofía. La literatura identifica (Saba 2005:143) casos judiciales de relevancia en esta dirección, en especial uno que tuvo importancia por su esclarecedora fundamentación. Se trata de un caso judicial iniciado por los padres de los alumnos varones del Colegio Montserrat de la Universidad Nacional de Córdoba, que fue siempre exclusivo para varones, llegan hasta la Corte Suprema de Justicia porque ese Colegio decidió permitir el ingreso de mujeres. El debate se centró en la constitucionalidad o no del sexo. La mayoría de los jueces centró su fundamento en la igualdad de oportunidades que se sustenta en la no discriminación e hizo lugar al pedido. Uno de los jueces centra su fundamento en la igualdad como no sometimiento y señala: "las categorías fundadas en el sexo no deben usarse para crear o perpetuar la inferioridad legal, social y económica de la mujer. En todo caso, las clasificaciones fundadas en el sexo pueden ser utilizadas para compensar a las mujeres por las inhabilidades que ellas han sufrido a través de la historia" (Caso González de Delgado, Cristina y otros c/Universidad Nacional de Córdoba).
} 
igualdades. Esta evidencia coloca al sistema político en una situación visible de irrazonabilidad y falta de capacidad para intervenir sobre los factores estructurales que afectan el desarrollo y la integración mediante un cambio en las pautas distributivas del crecimiento acompañado de un conjunto de políticas que, ancladas en derechos, intervengan sobre la dualidad social y fomenten un bienestar equitativo.

En esta realidad, la noción de igualdad en la arena política debería combinar los enfoques de posiciones, oportunidades y estructura. Las políticas de transferencias condicionadas de ingresos, tal como están definidas y diseñadas institucionalmente, son insuficientes como estrategia para enfrentar la vulnerabilidad, la pobreza y las desigualdades, por el contrario, no sólo frenan el proceso de igualdad de género sino que reproducen desigualdades cruzadas.

En síntesis, es impensable consolidar la democracia y el proceso de desarrollo sin que exista igualdad de género en nuestras sociedades porque tanto los hombres como las mujeres son los agentes que los protagonizan y los que lo harán posible, siempre y cuando caminen a la par.

\section{Referencias bibliográficas}

AMOROS, C. La gran diferencia y sus pequeñas consecuencias... Para la lucha de las mujeres. Madrid, Ediciones Cátedra, Colección Feminismos, Universidad de Valencia, 2007.

ANSES. Observatorio de la Seguridad Social. Buenos Aires, 2011. En línea: http:// observatorio.anses.gob.ar/files/subidas/AUH

ARCIDIÁCONO, P. La política del "mientras tanto". Programas sociales después de la crisis 2001-2002. Buenos Aires, Editorial Biblos, Colección Derechos Sociales y Políticas Públicas, 2012.

BARRIENTOS, A. "Dilemas de las políticas sociales latinoamericanas", en: Revista Nueva Sociedad. No 239, Buenos Aires, mayo-junio 2012.

BERLÍN, I. "Dos conceptos de libertad" en: Cuatro ensayos sobre la libertad. Madrid, Alianza Editorial, 1993. 
CEPAL. Informe de la reunión: Gobernabilidad democrática e igualdad de género en América Latina y el Caribe. Santiago de Chile, 27 y 28 de octubre de 2003.

CEPAL ¿Qué Estado para qué igualdad? (LC/G.2450 (CRM.11/3)). Santiago de Chile, 2010.

CEPAL. El salto de la autonomía. De los márgenes al centro. Santiago de Chile, 2011.

DUBET, F. "Los límites de la igualdad de oportunidades", en: Nueva Sociedad. Buenos Aires, Mayo-Junio 2012, No 239.

FALETTO, E. "Política Social, Desarrollo y Democracia en América Latina. Las funciones del Estado", en: Revista de Sociología. No 8, Facultad de Ciencias Sociales, Universidad de Chile. Chile, 1993.

FILGUEIRA, F. "El nuevo modelo de prestaciones sociales en América Latina: eficiencia, residualismo y ciudadanía estratificada”, en: BRIAN, R. (ed.) Ciudadanía y política social latinoamericana. San José, Flacso, 1998.

LAMAS, M. El género: la construcción cultural de la diferencia sexual. México, PUEGMiguel Angel Porrúa, 1996.

LEVÍN, S. "Déficit de democracia en la Argentina del siglo XXI. Las (des) igualdades de integración de integración social", en: Estructura social y problemas sociales en Argentina. II Encuentro de Cátedras de estructura social 2010. La Plata, Editorial Universidad Nacional de La Plata, 2012 (en prensa).

LEVÍN, S. "Un nuevo criterio de justicia en Argentina: aborto por violación" (en proceso de evaluación), en: Iconos. Revista de Ciencias Sociales $\mathrm{N}^{\circ}$ 45. Ecuador, FLACSO, 2012 (en prensa).

LOMELÍ, E. "Conditional Cash Transfers as Social Policy in Latin America: An Assessment of their Contributions and Limitations", en: Annual Review of Sociology $\mathrm{N}^{\circ}$ 34, EE.UU, 2008.

MIDAGLIA, C. "Un balance crítico de los programas sociales en América Latina. Entre el liberalismo y el retorno del Estado", en: en: Nueva Sociedad. Buenos Aires, MayoJunio 2012, No 239.

MILL, J. S. Sobre la libertad. Madrid, Alianza Editorial, 1993.

NACIONES UNIDAS. Informe de la Cuarta Conferencia Mundial sobre la Mujer, Beijing, 4 a 15 de septiembre (A/CONF.177/20/Rev.1). Nueva York. Publicación de las Naciones Unidas, 1995.

NACIONES UNIDAS. "Conferencia de Naciones Unidas sobre Desarrollo Sostenible. Río +20”. Río de Janeiro, Brasil, 20 al 22 de junio de 2012.

O’DONNELL, G. "Pobreza y desigualdad en América Latina: algunas reflexiones políticas”, en: TOKMAN, V. E. y O’DONNELL, G. (eds.). Pobreza y desigualdad en América Latina. Buenos Aires, Paidós, 1999. 
PAUTASSI, L. "El enfoque de derechos y la institucionalidad de las políticas sociales", en: ABRAMOVICH, V. y PAUTASSI, L. (compiladores). La revisión judicial de las politicas sociales. Buenos Aires, Editores del Puerto S.R.L., 2009.

PAUTASSI, L. Programas de transferencias condicionadas de ingresos ¿Quién pensó en el cuidado? La experiencia en Argentina. Santiago de Chile, CEPAL, 2009.

PAUTASSI, L. y ZIBECCHI, C. La provisión de cuidado y la superación de la pobreza infantil. Programas de transferencias condicionadas en Argentina y el papel de las organizaciones sociales y comunitarias. Santiago de Chile, CEPAL, Serie Políticas Sociales 159, 2010.

PNUD. La democracia en América Latina. Hacia una democracia de ciudadanos y ciudadanas. Buenos Aires, Aguilar, Taurus, Alfaguara S.A, 2004.

PNUD. Informe sobre Desarrollo Humano. "La cooperación internacional ante una encrucijada. Ayuda al desarrollo, comercio y seguridad en un mundo desigual". 2005.

PNUD. "Desafíos para la igualdad de género en Argentina”. 2008.

SABA, R. “(Des) igualdad estructural”, en: Revista Derecho y Humanidades. No 11 , Facultad de Derecho de la Universidad de Chile, 2005.

SALVIA, A. (editor). Barómetro de la Deuda Social Argentina. Serie del Bicentenario (2010-2016)/ Año 1. Estado de situación del desarrollo humano y social. Barreras estructurales y dualidades de la sociedad argentina en el primer Bicentenario. Observatorio de la Deuda Social Argentina, Pontificia Universidad Católica Argentina, 2011.

UNESCO. "La interacción entre Democracia y Desarrollo". Informe de Síntesis. Boutros Boutros-Ghali. París, Naciones Unidas, 2003.

SERRANO, C. "La política social en la globalización. Programas de protección en América Latina". Unidad de la Mujer, Proyecto Gobernabilidad e Igualdad de Género. Santiago de Chile, CEPAL, 2005. 
casos. En caso de ser publicado el artículo, el/los autor/es transfieren todos los derechos de autor a Cátedra Paralela, sin cuyo permiso expreso no podrá reproducirse ninguno de los materiales allí publicados. Asimismo, Cátedra Paralela asume los derechos para editar, publicar, reproducir, distribuir copias, preparar trabajos derivados en papel y/o electrónicos e incluir el artículo en índices nacionales e internacionales o bases de datos. Se solicita a los/as colaboradores/as la entrega de la nota expresa de originalidad y cesión de los derechos de autor/a. La Revista Cátedra Paralela no se hace responsable por los trabajos no publicados ni se obliga a mantener correspondencia con los/as autores/as.

\section{Modelo Nota de Autorización - Revista Cátedra Paralela}

Por la presente autorizo a la Revista Cátedra Paralela, a la publicación del artículo de mi autoría

.........en el $\mathrm{N}^{\circ}$....... del año ......, publicación de la Escuela de Trabajo Social de la UNR y el Colegio de profesionales de Trabajo Social de la $2^{a}$ Circunscripción de la Provincia de Santa Fe.

Se deja constancia de que no corresponde retribución pecuniaria derivada del derecho de autor.

LUGAR Y FECHA:

FIRMA Y ACLARACIÓN: 